\title{
Scalpel blade contamination and risk of postoperative surgical site infection following abdominal incisions in dogs
}

\author{
Christina G. Lioce ${ }^{1 *}$, Elizabeth C. Davis ${ }^{1}$, Julie W. Bennett ${ }^{1}$, Forrest I. Townsend ${ }^{1}$ and Christopher P. Bloch ${ }^{2}$
}

\begin{abstract}
Objective: This prospective observation sought to determine if scalpel blades used for abdominal skin incisions in dogs are a significant source of bacterial contamination, and if these blades should be changed prior to use in deeper dissection.

Results: Scalpel blades were swabbed for culture prior to skin incision as a control, and then again following ventral midline abdominal skin incision in a total of 75 dogs. Culture and sensitivity results were compared with review of medical records for any evidence of pre- or postoperative incisional surgical site infection/inflammation (SSI). Of the 75 blades swabbed after skin incision, only $2(2.7 \%)$ had positive culture results. Of the 69 patients that survived to suture removal, there was evidence of SSI in 6 patients (8.7\%), only one of which had a positive scalpel blade culture (16.7\%). Neither the use of postoperative antibiotics nor positive scalpel blade culture results were good predictors of whether a patient would develop a SSI. Results of this pilot study suggest that there is no bacteriological evidence to support the use of a separate blade for deep dissection in routine surgical procedures.
\end{abstract}

Keywords: Scalpel, Contamination, Surgical site infection

\section{Introduction}

Postoperative infections are an inherent risk of any surgical procedure despite preventative measures. Previously published rates of surgical site infections (SSI) in small animals have ranged from 3-10\% [1-4]. Risk factors associated with SSI include duration of surgery, gender, increasing body weight, dirty surgical site, antimicrobial prophylaxis, and use of propofol [1, 4]. Straw et al. [5] described that up to $20 \%$ of skin bacteria is not susceptible to disinfection, being a potential source of bacterial contamination leading to incisional infections.

Numerous human medical studies have implied that use of separate blades as a means of infection control is unnecessary [6-11]. However, surgical wound contamination and infection statistics from human patients may not serve as a correlate to canine surgery. To the author's

\footnotetext{
*Correspondence: cglioce@gmail.com

1 Surgery Department, New England Animal Medical Center, West Bridgewater, MA, USA

Full list of author information is available at the end of the article
}

knowledge, there is only one report in the veterinary literature that examined scalpel blade contamination with skin bacteria, which only included orthopedic and neurosurgical procedures in dogs but found that the skin blade does not add significantly to bacterial inoculum contaminating clean wounds [5]. While deep tissue contamination can have a tremendous impact on orthopedic and neurosurgical procedures, there is currently no information on scalpel blade contamination from ventral midline abdominal incisions in dogs. Therefore, evaluating risk of SSI in this common surgery is warranted, and adds evidence to previous reports.

The purpose of this study was to determine if scalpel blades used for ventral midline abdominal skin incisions are a significant source of bacterial contamination for deeper structures in dogs, and if they should be changed prior to deeper dissection. We hypothesized that there would be little to no contamination of the scalpel blades used for skin incision, which would also not correlate with postoperative SSIs, and therefore in order to prevent 
infection the scalpel blade does not need to be changed prior to deeper dissection.

\section{Main text \\ Materials and methods}

An informal survey of 43 veterinary surgeons was undertaken to evaluate incidence of changing blades intraoperatively and included reasons for changing blades. The survey was performed using an anonymous survey website (SurveyMonkey Inc, San Mateo, CA).

Canine patients undergoing ventral midline abdominal incisions longer than $10 \mathrm{~cm}$ were included in the study, regardless of procedure performed. Patients that did not survive to suture removal or 30 days postoperatively were excluded from analysis of SSI risks. Each patient was aseptically prepared using $2 \%$ chlorhexidine gluconate scrub and 70\% isopropyl alcohol according to standard recommended protocol [12]. The environmental conditions in the surgical suite were similar for each case. Each patient also received a prophylactic dose of either cefazolin $22 \mathrm{mg} / \mathrm{kg}$ IV or ampicillin/sulbactam $50 \mathrm{mg} / \mathrm{kg}$ IV within 30 min prior to skin incision.

Two samples were obtained from each patient. A sterile \#10 blade was placed on the instrument table, then swabbed with a sterile moistened culturette as the control sample prior to contact with skin. The surgical area was then isolated with four cloth drapes and overlying fenestrated drape, and the ventral midline abdominal incision was made with the \#10 blade. Immediately after skin incision, the \#10 scalpel blade was swabbed with another sterile moistened culturette as the study sample. The surgeon's assistant performed this sampling sterilely before contact with the patient's skin, and sampling of each side and along the edge of each blade (all that contacted the skin during incision) was performed. Each surgical procedure progressed accordingly, without changing instruments, gloves, or blades after sampling.
The microbiological sampling technique and testing was similar to that used in previous veterinary studies [13-15]. Submitted swabs were stored in Amies Clear gel preservation medium with sodium thioglycolate and planted on a Trypticase Soy Agar w/ 5\% sheep blood and a MacConkey agar plate. The culture plates were examined for growth at 24 and $48 \mathrm{~h}$, and if present the species was identified. If needed, a subculture was performed to blood agar and Mac agar respectively to isolate individual bacterial colonies. The plates were then held an additional $24 \mathrm{~h}$. If no changes were noted, the identification and quantity were reported out as final.

Data was collected from medical records and followup calls to primary veterinarians if needed, as detailed in Table 1. Inclusion criteria for evaluation of postoperative SSI were survival to suture removal, a positive bacterial culture of the incision, or a diagnosis and antimicrobial treatment by a veterinarian after visual assessment of purulent discharge, heat, redness, pain, or localized swelling of the incision. Patients were excluded from evaluation of postoperative SSI if they died or were euthanized prior to suture removal and not for reasons related to SSI, and if medical records or contact with their primary veterinarian did not provide information regarding incisional healing within 30 days postoperatively.

Statistical analyses were conducted using SPSS v.24 software. Significance was assessed at the level of $\alpha=0.05$. Logistic regression was used to model the effects of continuous variables (age, weight, anesthesia time, incision length) on the likelihood of a SSI. Fisher's Exact Test was used to evaluate associations between the frequencies of discrete variables (counts) and the frequency of SSI.

\section{Results}

Results of the informal survey of veterinary surgeons revealed that $36.1 \%$ of them change their scalpel blades after initial skin incision, as they feel the skin blade could

Table 1 Data variables collected for each patient and definitions of their reporting

\begin{tabular}{|c|c|}
\hline Variable & Definition \\
\hline Patient signalment & Age, sex, breed, weight \\
\hline Procedure performed & Clean vs. clean-contaminated vs. contaminated vs. dirty \\
\hline Duration of anesthesia & Time from induction to cessation of isoflurane administration, in minutes \\
\hline Perioperative antibiotic & Cefazolin 22 mg/kg IV or ampicillin/sulbactam 50 mg/kg IV \\
\hline Propofol & Used vs. not used, 4-6 mg/kg IV \\
\hline Preoperative skin condition & $\begin{array}{l}\text { Within normal limits vs. scrub irritation vs. previous incision (within } \\
30 \text { days) vs. pyoderma vs. dirty }\end{array}$ \\
\hline Incision length & Greater than or equal to $10 \mathrm{~cm}$ \\
\hline Control and study sample culture identifications & Positive vs. negative, isolate \\
\hline Postoperative evidence of SSI & As defined by the Center for Disease Control (CDC, Table 2) \\
\hline
\end{tabular}


Table 2 CDC criteria for defining surgical site infection [4]

\begin{tabular}{|c|c|c|c|}
\hline & Superficial incisional SSI & Deep incisional SSI & Organ/space SSI \\
\hline Timing & Within 30 days of surgery & $\begin{array}{l}\text { Within } 30 \text { days of surgery or } 1 \text { year if } \\
\text { implant in place }\end{array}$ & $\begin{array}{l}\text { Within } 30 \text { days of surgery or } 1 \text { year of } \\
\text { implant in place }\end{array}$ \\
\hline Location & $\begin{array}{l}\text { Only skin or subcutaneous tissues of } \\
\text { incision }\end{array}$ & $\begin{array}{l}\text { Deep soft tissues (fascia, muscle) of the } \\
\text { incision }\end{array}$ & $\begin{array}{l}\text { Any area other than the incision which was } \\
\text { opened or manipulated in surgery }\end{array}$ \\
\hline Clinical aspects $^{a}$ & $\begin{array}{l}\text { Purulent discharge } \\
\text { Organisms isolated from an aseptically } \\
\text { collected sample of fluid or tissue } \\
\text { One or more: pain/tenderness, localized } \\
\text { swelling, redness, heat, and incision is } \\
\text { deliberately opened by surgeon unless } \\
\text { culture negative }\end{array}$ & $\begin{array}{l}\text { Purulent drainage from deep incision but } \\
\text { not organ/space } \\
\text { Deep incision spontaneously dehisces or } \\
\text { is deliberately opened when patient has } \\
\text { one or more: fever, localized pain/ten- } \\
\text { derness unless culture negative } \\
\text { Abscess or other evidence of infection on } \\
\text { direct exam, during reoperative, or by } \\
\text { histopathology or radiology }\end{array}$ & $\begin{array}{l}\text { Purulent drainage from drain placed in } \\
\text { organ/space } \\
\text { Organisms isolated from aseptically col- } \\
\text { lected sample from organ/space } \\
\text { Abscess or other evidence of infection on } \\
\text { direct exam, during reoperation or by } \\
\text { histopathology or radiology } \\
\text { Diagnosis of organ/space SSI by attending } \\
\text { clinician }\end{array}$ \\
\hline
\end{tabular}

a One or more must be present

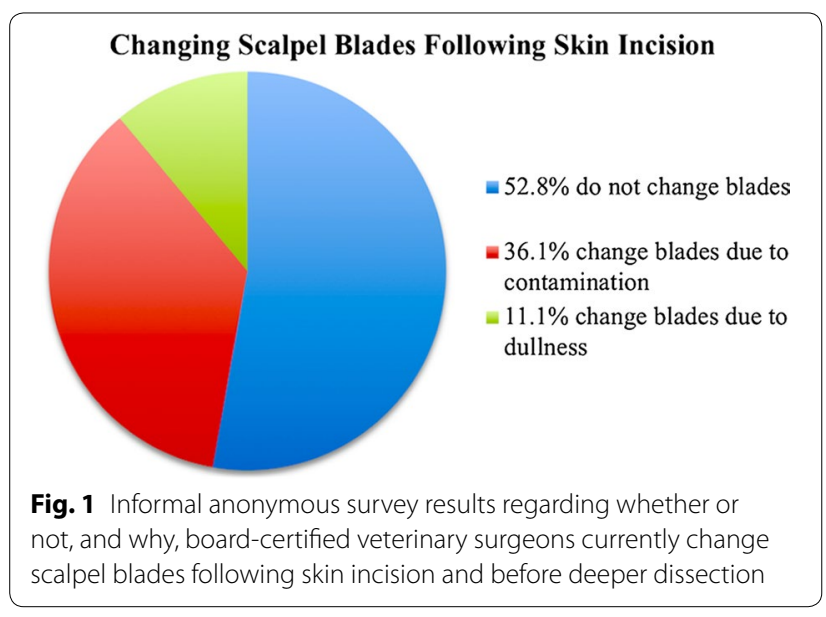

potentially introduce bacteria into the deeper surgical field (Fig. 1).

A total of 75 dogs that underwent ventral midline abdominal incisions were eligible for inclusion in the study. Of the 75 scalpel blades cultured after skin incision, there were only 2 that were positive for bacterial growth (2.7\%). One sample was positive for Acinetobacter sp., and that patient did not develop a postoperative SSI. The second positive blade culture grew Staphylococcus pseudintermedius; that patient had an erythemic previous spay incision present at the time of surgery and also went on to develop incisional erythema and purulent discharge. Unfortunately, the owners declined sampling the incision, so the presumed SSI was not confirmed via culture.

There was one positive control sample, which was a suspected laboratory/testing contaminant (Achromobacter sp.) rather than a true skin incision contaminant. Six dogs (8.0\%) died or were euthanized prior to suture removal for reasons unrelated to SSI (poor prognosis, decompensation). Of the 69 dogs that survived to suture removal, six (8.7\%) reportedly developed and were treated for a SSI but only two $(2.9 \%)$ were confirmed with positive culture results. One of those incisions was infected with Eschericia coli and Enteroccocus sp., and the other infected with Eschericia coli and Streptococcus sp. One of the diagnosed SSIs had a negative culture despite the presence of purulent discharge, and three did not have cultures performed as they were declined by the owners. All diagnosed SSIs were treated successfully with cephalexin, cefpodoxime, or amoxicillin/clavulanic acid.

Preoperative skin condition was recorded for every patient, and abnormalities were noted in 15 patients (20\%). Abnormalities noted were scrub irritation (8 cases, 10.7\%), previous healing or healed incision within 30 days postoperatively ( 2 cases, $2.7 \%$ ), pyoderma ( 2 cases, $2.7 \%$ ), and visible dirt/debris (3 cases, $4.0 \%)$. Preoperative skin condition was a poor predictor of whether a patient would experience a SSI. All four continuous variables were poor predictors of whether a patient would experience a SSI (Additional file 1: Table S1). None of the regression models were statistically significant $(\mathrm{P}>0.05)$, and model fit was poor $(<0.15)$ in all cases. There was no significant association between the frequency of SSI and any of the observed discrete variables $(\mathrm{P}>0.05$ in all cases; Additional file 1 Table S2).

\section{Discussion}

Bacterial contamination rate of scalpel blades used for ventral midline abdominal incision in dogs has not been previously reported. In the present study, only $2.7 \%$ of scalpel blades had positive cultures after incising through skin. The use of a chlorhexidine neutralization step was not included in microbiological testing, which may have significantly increased the number of false negative cultures. However, the purpose of this study was to evaluate 
the potential risk of contamination following a standard skin preparation protocol, which would not include a chlorhexidine neutralization step prior to further scalpel blade use after skin incision. Therefore, our results reflect the true clinical situation of abdominal surgical procedures.

The bacterial species isolated from the two positive scalpel blade cultures were Acinetobacter sp. and Staphylococcus pseudintermedius, both of which are common opportunistic skin pathogens of concern due to their high level of antimicrobial resistance [4]. Despite this concern, only one of those two dogs whose scalpel blades were positive for bacterial growth went on to develop a presumed SSI, which was unfortunately not confirmed via culture but responded to treatment with amoxicillin/clavulanic acid. This patient's blade cultured Staphylococcus pseudintermedius, and this dog had undergone a routine spay by their primary care veterinarian within 30 days of their surgery included in this study. On initial presentation, this previous spay scar already appeared subjectively inflamed, likely resulting in the positive bacterial culture of the scalpel blade used to make the abdominal incision over the scar. Based on these results, the scalpel blade used to incise skin during abdominal surgery is likely not a significant source of contamination but there may be increased risk of SSI if there is already evidence of inflammation or infection. Whether or not the skin scalpel blade is contaminated is not a good predictor of whether a patient will suffer a SSI, but if incising through active infection or SSI the scalpel blade likely should be changed and the affected tissue cultured or even excised prior to closure.

In the present study, there were six (8.7\%) postoperative SSIs diagnosed, which is higher than reported in more recent studies of SSI rates in small animal surgery. Despite previously reported rates of $3-10 \%$, a study by Turk et al. found that in 846 dogs undergoing various surgical procedures there were only $26(3.0 \%)$ identified SSIs [2]. They also found that hypotension, class of surgery, and use of an implant increased risk of SSI. A more recent large study of 1271 dogs and cats reported a low and very similar rate of SSI at $2.83 \%$ [16]. Further study of scalpel blade contamination and SSI risk with a larger number of cases is warranted.

\section{Conclusions}

Regardless of the level of care provided, postoperative infections are an inherent risk of surgery and will likely continue to be a source of investigation. The present study showed very low bacterial contamination on skin scalpel blades, however was underpowered to evaluate relationship to SSI.

\section{Limitations}

There were several limitations of the present study. Most notable was the relatively small sample size, leading to only two positive scalpel blade cultures $(2.7 \%)$ and six postoperative SSIs $(8.7 \%)$. This prevented any strong correlation of previously reported significant factors in development of SSI. Although logistic regression models correctly predicted presence or absence of an SSI in most cases, this was because each model predicted no SSI for every case, while SSIs were uncommon (6 of the 69 cases in which the patient survived long enough to assess). Power of analysis calculations showed that definitive statements regarding the results of this study require 10 each of positive scalpel blade cultures and diagnosed SSIs, however this study proves the low incidence of positive scalpel blade cultures out of 75 abdominal incisions and can preliminarily report their lack of significance in risk of postoperative SSI.

Another limitation of this study was that of the SSIs diagnosed by different veterinarians based on the CDC's definitions, only half actually had cultures performed at the time of diagnosis, and one of those cultures returned negative for bacterial growth despite purulent discharge being present. All of the diagnosed SSIs were treated successfully with antibiotics, empirically or based on culture results, but confirmation with a positive culture of the incision would have been ideal. Finally, a different method of scalpel blade sampling and testing (e.g., immersion in thioglycollate broth or sonication) for culture could potentially yield higher bacterial counts and different results. However, as stated before, swabbing of the scalpel blade areas that came in contact with the skin during incision was sufficient for the purposes of this study.

\section{Additional file}

Additional file 1: Table S1. Results of logistic regression analysis used to model the effects of continuous variables (age, weight, anesthesia time, incision length) on the likelihood of a SSI ${ }^{1}$. Table S2. Results of Fisher's Exact Tests to evaluate associations between SSI frequency and discrete variables.

Abbreviation

SSI: surgical site infection.

Acknowledgements

Not applicable

\section{Authors' contributions}

$\mathrm{CL}$ conducted the research and wrote the manuscript, ED designed the study and contributed to writing the manuscript, JB and FT contributed to writing the manuscript, and CB analyzed and interpreted the data. All authors read and approved the final manuscript. 


\section{Funding}

Funding was provided by the New England Animal Medical Center as part of residency research funds, with no role in design of the study and collection, analysis, and interpretation of data and in writing the manuscript.

\section{Availability of data and materials}

The datasets used and/or analyzed during the current study are available from the corresponding author on reasonable request.

\section{Ethics approval and consent to participate}

This study was conducted in compliance with both the principles of the Basal Declaration as well as institutional guidelines, although the institution lacked an available ethics committee. This study involved client-owned animals; informed written consent from all canine owners was obtained for the surgical procedures performed and informed verbal consent for inclusion in the study, as practices adhered to a high standard of veterinary care and did not involve any invasive testing.

\section{Consent for publication}

Not applicable.

\section{Competing interests}

The authors declare that they have no competing interests.

\section{Author details}

${ }^{1}$ Surgery Department, New England Animal Medical Center, West Bridgewater, MA, USA. ${ }^{2}$ Department of Biological Sciences, Bridgewater State University, Bridgewater, MA, USA.

Received: 6 May 2019 Accepted: 17 July 2019

Published online: 25 July 2019

\section{References}

1. Eugster S, Schawalder P, Gaschen F, Boerlin P. A prospective study of postoperative surgical site infections in dogs and cats. Vet Surg. 2004;33:542-50.

2. Turk R, Singh A, Weese JS. Prospective surgical site infection surveillance in dogs. Vet Surg. 2015:44:2-8.
3. Vasseur PB, Levy J, Down E, Eliot J. Surgical wound infection rates in dogs and cats. Vet Surg. 1998;17(2):60-4.

4. Weese JS. A review of postoperative infections in veterinary orthopaedic surgery. Vet Comp Orthop Traumatol. 2008;21:99-105.

5. Straw RC, Tomlinson JL, Fales WH. Scalpel blade contamination with skin bacteria during orthopedic and neurosurgical procedures in dogs. Vet Surg. 1987;16:25-30.

6. Cibella VB, Smith L, Haas M. Skin blade versus deep blade: a vehicle of contamination in podiatric surgery. J Foot Surg. 1990;29:44-5.

7. Fairclough JA, Mackie IG, Mintoet-czyz W, Phillips GE. The contaminated skin knife, a surgical myth. J Bone Joint Surg. 1983;83:210.

8. Hill R, Blair S, Neely J, Ramanathan M. Changing knives a wasteful and unnecessary ritual. Ann R Coll Surg Engl. 1985;67:149-51.

9. Jacobs HB. Skin knife-deep knife. The ritual and practice of skin incisions. Ann Surg. 1972;179:102-4.

10. Ramon R, Garcia S, Combalia A, Puig de la Bellacasa J, Segur JM. Bacterial study of surgical kinves: is the use of two blades necessary? Arch Orthop Trauma Surg. 1994;1994(113):157-8.

11. Schindler OS, Spencer RF, Smith MD. Should we use a separate knife for the skin? J Bone Joint Surg. 2006;88:382-5.

12. Davidson JR, Burba DJ. Surgical instruments and aseptic technique. In: Bassert JM, McCurnin DM, editors. McCurnin's Clinical textbook for veterinary technicians. 7th ed. St. Louis: Saunders Elsevier; 2009. p. 971-972.

13. Gibson KL, Donald AW, Harihan H, McCarville C. Comparison of two presurgical skin preparation techniques. Can J Vet Res. 1997;61:154-6.

14. Tannahill VJ, Cogan T, Allen K, Acutt E, Busschers E. Efficacy and dermal tolerance of a novel alcohol-based skin antiseptic in horses. Vet Surg. 2018;47:572-7.

15. Zubrod CJ, Farnsworth KD, Oaks JL. Evaluation of arthrocentesis site bacterial flora before and after 4 methods of preparation in horses with and without evidence of skin contamination. Vet Surg. 2004;33:525-30.

16. Stickney DG, Mankin KT. The impact of postdischarge surveillance on surgical site infection diagnosis. Vet Surg. 2018:47:66-73.

\section{Publisher's Note}

Springer Nature remains neutral with regard to jurisdictional claims in published maps and institutional affiliations.
Ready to submit your research? Choose BMC and benefit from:

- fast, convenient online submission

- thorough peer review by experienced researchers in your field

- rapid publication on acceptance

- support for research data, including large and complex data types

- gold Open Access which fosters wider collaboration and increased citations

- maximum visibility for your research: over $100 \mathrm{M}$ website views per year

At BMC, research is always in progress.

Learn more biomedcentral.com/submissions 